\title{
FIXED POINT THEORY IN COMLEX VALUED METRIC SPACE
}

\author{
Abhishek Koundal \\ Department of Mathematics \\ Email ID:akmonu98@gmail.com \\ Chandigarh University, Mohali, Punjab
}

\begin{abstract}
The aim of this paper is to establish and prove several results on common fixed point for a pair of mappings satisfying more general contraction conditions portrayed by rational expressions having point-dependentcontrolfunctionsascoefficientsincomplexvaluedmetricspaces.Fixedpointtheory in complex valued metric space using contractive conditions, rational inequality, common limit range property for two pairs of mapping deriving common fixed-point results under a generalized altering distance functions, E.A and CLR property.Obtaining consecutive approximations to the fixed point of an approximate mapping is the goal of a variety of processes in numerical analysis and approximation theory. Our goal in this paper is to examine fixed point theory and its applications in metric spaces, as well as to develop several fixed-point theorems in entire metric spaces that generalize many renowned mathematicians' achievements.
\end{abstract}

Keywords: Fixed-point theory, Metric space, Complete Metric space, Continuous function.

\section{INTRODUCTION}

Azam et al. [1] introduced the concept of complex valued metric space by establishing a fixed-point result for mapping in complex valued metric space that fulfils a reasonable inequality. Several works have been published since then that deal with the fixed point hypothesis in complex valued metric space (see [3-11]and references in that) Fixed-point was first concentrated by Rao et al. [12].

findings for various mappings that satisfy a rational requirement in complex valued b-metric spaces (see [13-16] and the relevant references in. Sintunavaratel al. (9,10), Sitthikul and Saejung [11], and Singhetal.[8] have recently established fundamental fixed-point results by substituting the consistency of contractive condition to control functions in complex valued metric spaces. Inacontinuationof[8,11,15,17],somenormalfixed-point result for a couple of mapping fulfilling more broad contractive conditions including rational expression having point-subordinate control function as coefficients in complex valued b-metric spaces have been proved by manyauthors.

The well-known Banach contraction principal states that "If $\mathrm{X}$ is complete metric space and $\mathrm{f}$ is a contractionmappingonXintoitself,thenfhasuniquefixedpointinX”. Manymathematiciansworked on this principal. Kannan proved that If $\mathrm{T}$ is self-mapping of a complete metric space $\mathrm{X}$ into itself satisfying:

$\mathrm{d}(\mathrm{Tx}, \mathrm{Ty}) \leq[\mathrm{d}(\mathrm{Tx}, \mathrm{x})+\mathrm{d}(\mathrm{Ty}, \mathrm{y})]$ forall $\mathrm{x}, \mathrm{y} \in \mathrm{X}$, where $\alpha \in \in 0,{ }^{1} \emptyset$.

Then $\mathrm{T}$ has unique fixed point in $\mathrm{x}$.

Fisher, proved the result with

$\mathrm{d}(\mathrm{Tx}, \mathrm{Ty}) \leq[\mathrm{d}(\mathrm{Ty}, \mathrm{x})+\mathrm{d}(\mathrm{Tx}, \mathrm{y})]$ forall $\mathrm{x}, \mathrm{y} \in X X, w w h$ reeeee $\alpha \in \emptyset 0, \stackrel{1}{\uparrow} \emptyset$. 
A similar conclusion was also obtained by Chatterjee

In 1977, the mathematician Jaggi, introduced the rational expression first time as:

$\mathrm{d}(\mathrm{Tx}, \mathrm{Ty}) \leq \alpha \alpha \alpha \alpha(x x, y y)+\beta \beta^{\operatorname{dd}(x x, T T x x) \operatorname{dd}(y y, T T y y)} \frac{\operatorname{defffeenaan}}{\operatorname{dd}(x x, y)} \quad x x, y y \in X X, x x \neq y y, 0 \leq \alpha \alpha+\beta \beta<1$.

In 1980 the mathematician Jaggi and Das obtained some fixed-point theorems with the mapping satisfying:

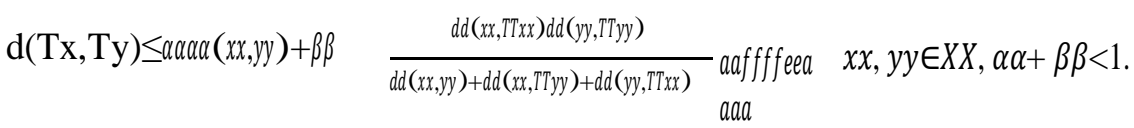

In the present paper we also finding a new rational expression, using complete metric spaces, which satisfy the many result of great mathematicians.

\section{Preliminaries}

Banach fixed point theorem [1] in complete metric space has been summed up in numerous spaces. In2011Azametal.[2]presentedthethoughtofcomplex-valuedmetricspaceandbuilt-upsufficient condition for the presence of common fixed point of a pair of mappings fulfilling a contractive condition. The possibility of complex-valued metric spaces can be abused to define complex-valued normed spaces and complex-valued Hilbert spaces, moreover it offers various research exercise in numerical examination. The theorems demonstrated by Azam et al. [2] and Bhatt et al. [18] utilizes the rational in equality in a complex valued metric space as contractive condition. In this paper. We presenttheideaofproperty(E.A)incomplex-valuedmetricspace,todemonstratesomenormalfixed-

pointresultforafourfoldofself-mappingfulfillingacontractiveconditionof'max'type.Ouroutcomes sum up different theorems of customary metricspaces.

A real valued function from a set $X X$ into $R$ is an ordinary metric $d$, where $X$ is a non-empty set. $d: X$ $\mathrm{X} \mathrm{R}$ is the code. A complex number $\mathrm{zC}$ is an ordered pair of real numbers with $\operatorname{Re}(\mathrm{z})$ as the first coordinate and Im as the second co-ordinate (z). As a result, a complex valued metric $\mathrm{d}$ is a function from a set $\mathrm{XX}$ into $\mathrm{C}$, where $\mathrm{X}$ is a nonempty set and $\mathrm{C}$ is the complex number set.

That is, $\mathrm{d}: \mathrm{X} \times \mathrm{X} \rightarrow \mathrm{C}$.

let $\mathrm{z}_{1, \mathrm{z}} \in \mathrm{C}$, define a partial order on $\mathrm{C}$ as follows:

$\mathrm{z} 1 \leq \mathrm{z} 2$ if and only if $\operatorname{Re}\left(\mathrm{z}_{1}\right) \leq \operatorname{Re}\left(\mathrm{z}_{2}\right), \operatorname{Im}\left(\mathrm{z}_{1}\right) \leq \operatorname{Im}\left(\mathrm{z}_{2}\right)$

Itfollowsthatz $\leq_{1} \leq \mathrm{z}_{2}$ ifoneofthefollowingconditionsissatisfied:

$\operatorname{Re}\left(\mathrm{z}_{1}\right)=\operatorname{Re}\left(\mathrm{z}_{2}\right), \operatorname{Im}\left(\mathrm{z}_{1}\right)<\operatorname{Im}\left(\mathrm{z}_{2}\right)$

$\operatorname{Re}\left(\mathrm{z}_{1}\right)<\operatorname{Re}\left(\mathrm{z}_{2}\right), \operatorname{Im}\left(\mathrm{z}_{1}\right)=\operatorname{Im}\left(\mathrm{z}_{2}\right)$

$\operatorname{Re}\left(\mathrm{z}_{1}\right)<\operatorname{Re}(\mathrm{z} 2), \operatorname{Im}\left(\mathrm{z}_{1}\right)<\operatorname{Im}\left(\mathrm{z}_{2}\right)$

$\operatorname{Re}\left(\mathrm{z}_{1}\right)=\operatorname{Re}(\mathrm{z} 2), \operatorname{Im}\left(\mathrm{z}_{1}\right)=\operatorname{Im}\left(\mathrm{z}_{2}\right)$.

In(1),(2)and (3), we have $\left|z_{1}\right|<\left|z_{2}\right|$. In (4), we have $\left|z_{1}\right|=\left|z_{2}\right|$.In particular, $z_{1}<z_{2}$ if $z_{1} \neq z_{2}$ and one of (1),(2),(3) is satisfy. In this case $\left|z_{1}\right|<\left|z_{2}\right|$.We will write $z_{1}<z_{2}$ if only (3) satisfy. Further,

$0 \leq \mathrm{z}_{1}<\mathrm{z}_{2} \rightarrow\left|\mathrm{z}_{1}\right|<\left|\mathrm{z}_{2}\right|$ 
$\mathrm{z}_{1} \leq \mathrm{z}_{2}$ and $\mathrm{z}_{2}<\mathrm{z}_{1} \rightarrow \mathrm{z}_{3}$

Azam et al. [2] defined the complex valued metric space (X,d) in the following ways:

Defination1.1 Let $x$ be a non-empty set. Suppose that the mapping $d: X \times X \rightarrow C$ satisfies the following conditions:

(C1) $0 \leq \mathrm{d}(\mathrm{x}, \mathrm{y})$ for all $\mathrm{x}, \mathrm{y} \mathrm{X}$ and $\mathrm{d}(\mathrm{x}, \mathrm{y})=0$ if and only if $\mathrm{x}=\mathrm{y}$;

(C2) $d(x, y)=d(y, x)$ for all $x, y \varepsilon X$;

(C3) $d(x, y) \leq d(x, z)+d(z, y)$ for all $x, y, z^{\varepsilon} \varepsilon X$

Then $\mathrm{d}$ is called a complex valued metric on $\mathrm{X}$, and $(\mathrm{X}, \mathrm{d})$ is called a complex valued metric space.

\section{Common Fixed point Theorems Using Property (E.A) in complex valued Metric} spaces. Fixed Point Theorem (E.A) Property [19]

In this paper author proved some important fixed-point theorems using (E.A) property and (CLR) property in complex valued metric space in which the author also used the notion of partial order.

Theorem[a] Let $(\mathrm{X}, \mathrm{d})$ be a complex valued metric space and A, B, S, T: X $\rightarrow X$ be four self-mapping satisfying.

(i). $A(X) \subseteq T(X), B(X \subseteq) S(X)$,

(ii). d(Ax, By) <k max (d (Sx, Ty), d (By, Sx), d (By, Ty), $\forall x$,

$\mathrm{y} \in \mathrm{X}, 0<\mathrm{k}<1$, (iii). The pairs (A, S) and (B, T) are

weaklycompatible,

(iv). One of the pair (A, S) and (B, T) satisfy(E.A)

If the range of one of the mapping $\mathrm{S}(\mathrm{X})$ or $\mathrm{T}(\mathrm{X})$ ids complete subspace of Then mappings $\mathrm{A}, \mathrm{B}, \mathrm{S}$ and $\mathrm{T}$ have unique common fixed point in $\mathrm{X}$.

\section{Fixed Point Theorem Using (CLR)-Property}

The notion of (CLR)-property was defined by Sintunavarat and Kumam [20] in a metric space for a pair of self-mapping, which have the common limit in the range of one of the mappings.

Definition: (The (CLR)-property [20]) Suppose that (X, d) is a metric space and $f, g ; X \rightarrow X$. Two mappingfandgaresaidtosatisfythecommonlimitintherangeofgpropertyiflllllf $\mathrm{x}_{\mathrm{n}}=\operatorname{llllllg}_{n n \rightarrow \infty} \mathrm{x}_{\mathrm{n}}=\mathrm{gx}_{{ }_{n n \rightarrow \infty}}$ for some $\mathrm{x} \in \mathrm{X}$.

InthecomplexvaluedmetricspacethedefinitionwillbesamebutthespaceXwillbeacomplexvalued metricspace.

Theorem[b]. Let (X, d) be a complex valued metric space and A, B, S, T; X $\rightarrow X$ be a four selfmapping satisfying:

$$
\text { (i). } A(X) \subseteq T(X) \text {, }
$$


(ii). d (Ax, By) $\leq k \max (\mathrm{d}(\mathrm{Sx}, \mathrm{Ty}), \mathrm{d}(\mathrm{By}, \mathrm{Ty})) \forall \mathrm{x}$,

$\mathrm{y} \in \mathrm{X}, 0<\mathrm{k}<1$, (iii). The pairs (A, S) and (B, T) are

weaklycompatible.

If the pair $(A, S)$ satisfy $\left(C L R_{A}\right)$ property, or the pair $(B, T)$ satisfy $\left(C L R_{B}\right)$ property, then mapping $\wedge, \in: X \rightarrow[0,1)$ such that for all $\mathrm{x}, \mathrm{y} \in \mathrm{X}$ :

$\Lambda(\mathrm{Sx}) \leq \Lambda(\mathrm{x})$ and $\mathrm{E}(\mathrm{Sx}) \leq \mathrm{E}(\mathrm{x})$;

$\Lambda(T x) \leq \Lambda(x)$ and $E(T x) \leq E(x) ;$

$(\bigwedge+E)(x)<1$;

$\mathrm{d}(\mathrm{Sx}, \mathrm{Sy}) \preccurlyeq \Lambda(\mathrm{x}) \mathrm{d}(\mathrm{x}, \mathrm{y})+\underline{\underline{E}(x x) d d(x x, S x x) d d(y, T T y y)}$

$$
1+\mathrm{d}(\mathrm{x}, \mathrm{y})
$$

Then $\mathrm{S}$ and $\mathrm{T}$ have unique fixed point.

(iii). Six Maps with A common Fixed point in Complex valued Metric Spaces[22]

In this paper, author attained a common fixed-point theorem for six maps in complete valued metric space which is basically the generalization of [18]

Theorem: Let (X, d) be a complex valued metric space and F, G, I, J, K, L be self-maps of X satisfying the following conditions:

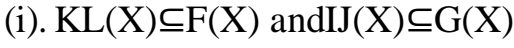

(ii). D(IJx,KLy) $\leq a d(F x, G y)+b(d(F x, I J x)+d(g y, K L y))+c(d(F x, K L y)+d(G y, I J x))$ for all $x, y \in X$, where $a, b, c \geq 0$ and $\mathrm{a}+2 \mathrm{~b}+2 \mathrm{c}<1$. Assumetatthepair(KL,G)and(IJ,F)areweaklycompatible,pair(K,L),(K,G),(L,G),(I,J),

(I,F)and(J,F)arecommutingpairofmaps.ThenK,L,I,J,GandFhaveuniquecommonfixedpointin X.

(iv). Some Common Fixed-Point Result for Rational Type Contraction Mapping in Complex valued Metric Space [23]

In this paper author demonstrate some fixed-point theorem for two pairs which fulfils a rational type condition in complex valued metric space.

\section{Fixed Point Theorem using E.A property}

Theorem: Let $(\mathrm{X}, \mathrm{d})$ be a complex valued metric space and A, B, S, T: $\rightarrow \mathrm{X}$ for self-mappings satisfying the following conditions:

(i). $A(X) \subseteq T(X), B(X) \subseteq S(X)$

(ii) For all $\mathrm{x}, \mathrm{y} \in \mathrm{X}$ and $0<\mathrm{a}<1$

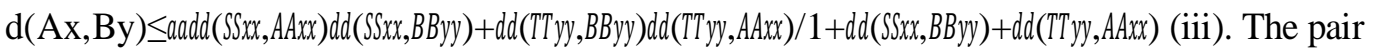

(A, S) and (B, T) are weakly compatible. 
(iv). One of the pair (A, S) or (B, T) satisfies (E.A)-property,

IftherangeS(X)orT(X)isclosedsubspaceofX,thenthemappingsA,B,SandThaveuniquecommon fixed point inX.

Fixed point theorem using (CLR)-property

IftherangeS(X)orT(X)idsclosedsubspaceofXthenthemappingsA,B,SandThaveuniquecommon fixed point inX.

\section{ACKNOWLEDGEMENT}

I will be very thankful to my professor, Dr. Sangeeta kumari to help me for motivation on research field.

\section{$\underline{\text { Conclusion }}$}

I will be very thankful to my professor, Dr. Sangeeta kumari to help me for motivation on research field.

It is concluded that metrics have very important role in the field of fixed-point theory in higher mathematics. Also, we have compared the continuity or uniform continuity in metric space with conversion and point wise convergence.

\section{References}

[1] S. Banach, Sür les op'erations dans les ensembles abstraits et leur application aux equations int'egrales, Fund. Math. 3 (1922)133-181.

[2] A. Azam, B. Fisher, M. Khan, Common fixed-point theorem in complexvalued metricspace.

[3]C.Klin-eamandC.Suanoom,“Somecommonfixed-

pointtheoremsforgeneralizedcontractivetypemappingsoncomplexvaluedmetricspaces,”AbstractandA ppliedAnalysis,vol.2013, ArticleID604215,6pages,2013.

[4] M. A. Kutbi, A. Azam, J. Ahmad, and C. Di Bari, "Some Common coupled fixed point results for generalized contractionin complex valuedmetricspaces,”JournalofAppliedMathematics, vol.2013,ArticleID352927,10pages,2013.

[5] H.K.Nashine,M.Imdad,andM.Hasan, “Commonfixedpointtheoremsunderrational contractions in complex valued metric spaces,” Journal of Nonlinear Science and Its Applications,vol.7, no.1,pp.4250,2014 .

[6] H.K. NashineandB. Fisher, "Common fixed point theorems for Generalized contraction involving rational expressions in complex valued metric spaces,” AnaleleStiintifice ale UniversitatiiOvidiusConstanta,vol.23,no.2,pp.179-185,2015.

[7] F. Rouzkard and M. Imdad, "Some common fixed point theorems on complex valued metric spaces,” Computers \&MathematicswithApplications,vol.64,no.6,pp.1866-1874,2012. 
[8] N.Singh,D.Singh,A.Badal,andV.Joshi,"Fixedpointtheorems in complex valued metric spaces,” Journal of the EgyptianMathematicalSociety,vol.24,no.3,pp.402-409,2016.

[9] W. Sintunavarat and P. Kumam, "Generalized common fixed point theorems in complex valued metric spaces and applications,”JournalofInequalitiesandApplications,vol.2012,article

84,12pages,2012.

[10] W.Sintunavarat,Y.J.Cho,andP.Kumam, “Urysohnintegralequationsapproachbycommonfixed points in complex-valued metric spaces,” Advancesin

Difference Equations, vol. 2013, article49,14pages,2013.

[11]K.SitthikulandS.Saejung, “Somefixedpointtheoremsincomplexvaluedmetricspaces,”FixedPointTh e oryandApplications, vol.2012,article189,11pages,2012.

[12] K. P. R. Rao, P. R. Swamy, and J. R. Prasad, "A common fixed point theorem in complex valued b- metric spaces,” Bulletin of Mathematics and Statistics Research, vol.1,no.1,pp.1-8,2013.

[13]A.K.Dubey,R.Shukla,andR.P.Dubey,“Somefixedpointtheoremsincomplexvaluedbmetricspaces,”Jo urnalofComplex Systems,vol.2015,ArticleID832467,7pages,2015.

[14]A.K. Dubey, “Common fixed point results for contractive mapping sin complex valued b-metric spaces,” Non-linear Functional AnalysisandApplications,vol.20,no.2,pp.257-268,2015.

[15] A.K. Dubey, R.Shukla, and R.P. Dubey, "Some common fixed point theorems for contractive mapping sin complex valued b-metric spaces,” Asian Journal of Mathematics and Apllications, vol.2015,ArticleIDama0266,13pages,2015.

[16] A. A. Mukheimer, "Some common fixed point theorems in complex valued b-metric spaces," The Scientific World Journal,vol.2014,ArticleID587825,6pages,2014.

[17] B. K. Dass and S. Gupta, "An extension of Banach contraction principle through rational expression,” Indian Journal of PureandAppliedMathematics,vol.6,no.12,pp.1455-1458,1975.

[18] S. Bhatt, S, Chaukiyal, R.C. Dimri, A common fixed point theorem for weakly compatible maps in complex-valued metric spaces, Int. J. Math. Sci. Appl. 1 (3) (2011)1385-1389.

[19]R.K Verma , H.K Pathak "Common fixed point theorems using(E.A)-property in complex valued metric space”,vol.11,347-355(2012).

[20] W. Sintunavarat, P. Kumam, "Common fixed point theorem for a pair of weakly compatible mappings in fuzzy metric space”, J. Appl. Math., Vol. 2011 (2011), Article ID 637958, 14pages

[21] Dass,Bk ,Gupta , S:An extension of Banach Contraction Principle through rational expression.Indian.J.Pure.Appl.Math6(12),1455-1458(1975)

[22] Rahul Tiwari,D.P. Shukla "Six maps with common fixed point in complex valued metric spaces “,vol.02,May(2012),827-832. 
[23] SumitChandok,Deepak Kumar "some common fixed point results for rational type contraction mappings in complex valued metricspaces,vol.2013ArticleID813707,6pages

[24]. BANACH, S(1922) :- Surles operation dans les ensembles abstracts etleur application aux equations integrals.Fun.Math,Vol.3,pp 133 - 181.

[25]CHATTERJEE,S.K(1972):-Fixedpointtheorems.Comptes.Rend.Acad.BulgariaSci.Vol.25,pp727730.

[26]. FISHER, B(1976):- A fixed point theorem for compact metric space Publ. Inst Math, Vol.25, 193194.

[27]. KANNAN, R(1969) :- Some results on fixed points -II .Bulletin of Calcutta Math.Soc.Vol.60,pp71- 76.

[28]. REICH; S (1971):- Some remarks concerning contraction mappings. Canada Math. Bull.Vol.1,pp 121-124 